\title{
The Impact of using English Medium Networks Breaking News on Enriching EFL Learners'Vocabulary. A Case Study of the Saudi University Students
}

\author{
Abdulghani Eissa Tour Mohammed \\ College of Sciences and Arts, Araas, Qussim University \\ Kingdom of Saudi Arabia, Qussim - Araas \\ Abdalgani71@yahoo.com
}

Ahmed Abdalla Saeed Adam

College of Arts and Science, Al-Baha University

Al-Mikhwa, Saudi Arabia

ahmad_saeed604@yahoo.com

\begin{abstract}
The current study attempts at investigating how TV breaking news impact on enriching EFL students' vocabulary. To undertake this study the researchers have randomly selected two groups of EFL students from AL-Qussim University college of Arts and Science, Arrass.The subjects were assigned to two groups in the experiment. To collect the required data a test was conducted and distributed among a sample of undergraduate EFL students from the college mentioned above during the academic year 2015-2016. The sample of the study consisted of (50) EFL students. A video clip of selective vocabulary focused on flood as a supportive material was given to the experimental group in which a variety of vocabulary list of target words was selected from the video clips. On the other hand, the control group was not accessed to the video clip. Then, a pre-test was given to the two groups. The first set was used as a pilot. Additionally, a post-test was then given to both groups and the data were collected and analyzed. The results of the study show that being access to English TV breaking news facilitates enriching vocabulary building. Moreover, it shows that using English network channels in classrooms raise the learning motivation of the students.
\end{abstract}

Keywords: Breaking news, English network channels, vocabulary building, video clip.

\section{INTRODUCTION}

Most of the related studies have pointed out on the role of TV news in enriching EFL students' English vocabulary generally. This study is attempting to view vocabulary enrichment through TV breaking news in particular. However, the researchers believe that reviewing a variety of vocabulary building approaches is extremely important for the purpose of the study.

Today, it possible for EFL learners as well as teachers to be access to different technological tools, in terms of advances satellites, digital video and broadband technology easily and effectively. According to (Cross, 2009) such a revolutionary tools have brought about a notable rise in the number of dedicated news channels and on-line news websites.

News services are undoubtedly bring a wealth of authentic videotexts that are a dynamic cultural, linguistic and educational resource ripe for exploitation within second and foreign language classrooms, self-access centers and home study environments to develop learners' 'real world' listening ability. In fact audio visual has become a necessity to EFL teachers and students alike rather than entertainment means of today. Teaching EFL through multimedia technology has been growing during the last decades internationally. Therefore, Saudi Arabia is not an exception in this global village where multimedia technology has become a part of peoples' daily life. Some studies showed that teachers in the past decade are used to teaching with multimedia technology including the Internet, TV and CD-ROMs as they believe that it can help students integrate real-life situations with the target language into the language classrooms. Although some people may think that watching TV is a mind-numbing process, some teachers still encourage students to learn English through watching TV news. More importantly, TV news can facilitate a way of lifelong learning for students, something 
that students can achieve when they are on their own. Education must not stop once the student leaves school. It must become a lifelong process.

\section{Statement of the Research Problem}

Throughout teaching university students it is has been observed that EFL learners have problems in learning and acquiring new vocabulary. This problem may be attributed to the methods of teaching vocabulary or to the materials that are used in developing EFL learners' vocabulary. The failure of learning vocabulary affects directly on other language learning skills; therefore, it is attempted to test using the method of English network breaking news as a tool to enhance students' vocabulary learning.

\section{Questions of the Study}

1. To what extentdo the English Medium Networks breaking News enrich EFL learners' vocabulary?

2. To what extent EFL learners interact with the network breaking news?

\section{Hypotheses}

1. The English medium network breaking news help enhance EFL learners' vocabulary building.

2. EFL learners positively interact with the breaking news and they are motivated to learn new vocabulary.

\section{LITERATURE REVIEW}

As stated above vocabulary building is one of the objectives of this study. Accordingly, the following section will include some related strategies that are found essential in building EFL students' vocabulary. Academic vocabulary will be addressed carefully in this study. Studies have shown that academic vocabulary differs from content vocabulary or 'traditional' models of vocabulary as it is not just content words such as import or export; rather, it is the "lifeblood of learning in all classes" (Zwiers, 2008 p. 10). Academic vocabulary incorporates words as mentioned earlier from Coxhead's Academic List (2000) such as conclusion, enable, and generate. It also combines vocabulary with the grammatical constructions, pragmatics, and word phrases that support explicit communication of ideas.

McCarthy (1990) claims that 'No matter how well the student learns grammar, no matter how successfully the sounds of L2 (second language learner) are mastered, without words to express a wider range of meanings, communication in L2 just cannot happen in any meaningful way.' Vocabulary is an essential part of language. Without adequate vocabulary knowledge, a second language learner will find it hard to do well in the four skills - speaking, listening, reading and writing. Read (2000) stressed the importance of vocabulary learning in second language acquisition. Unlike native speakers, second language learners (L2) go through a more conscious and demanding process of acquisition of vocabulary. They experience lexical gaps, the words they read which they simply do not understand or concepts that they cannot express as adequately as they could in the ir first language (L1). Many learners see second language learning as essentially a matter of learning vocabulary, so they devote a great deal of time to memorizing lists of L2 words. Language teachers and applied linguistic researchers therefore generally recognize the importance of vocabulary learning and are searching for the best ways of promoting it more effectively. Thus, from various points of view, vocabulary can be seen as a priority area in language learning.

In fact, many researchers such as Harley (1996) believe vocabulary learning to be a vital part of each student's life. On the same trend, O'Rourke (1974) points out that vocabulary affects students' thoughts, actions, aspirations, and success, especially in academic achievement. The relationship between vocabulary knowledge and reading comprehension is neither simple nor clear-cut. Some current LI reading theories suggest that processing at the word level is central to successful reading (Carr \& Levy, 1990; Rayner \& Pollatsek, 1989) (i.e., there may be a causal connection between vocabulary knowledge and reading comprehension).

It is also suggested that good readers not only comprehend more but also know more words and learn new words more easily (Bernhardt, 1991). In L2 research, while Grabe (1991) suggests that "virtually all second language reading researchers agree that vocabulary development is a critical component of reading comprehension." Therefore, it will be very helpful for English learners if they know the 
The Impact of Using English Medium Networks Breaking News on Enriching EFL Learners' Vocabulary. A Case Study of the Saudi University Students

most effective way to learn vocabulary. Shrum and Glisan (1994) provided many pedagogical methods for foreign language learning. In developing vocabulary skills, they expressed the view that new vocabulary should be introduced in a context using familiar vocabulary and grammar. In order to learn new words more effectively, they recommended the use of visuals for introducing new vocabulary. However, they were not specific as to whether motion or still visual would be more effective in introducing new vocabulary.

Still, one thing that is certain is that some concepts and actions are less likely to be understood with still visual images. Additionally, we can learn a lot ofup-to-date vocabulary via internet. It is found that the Internet provides a platform for students to get a taste of learning English through TV news. On the Internet, students can access the local news archive and watch English TV news. According to Paul (2000), we learn new words as we hear the words from the context of sentences and use the linguistic context to figure out what they mean. It also explains why children can learn a thousand words in a year. It is only possible through the linguistic context. (Sternberg, 1987) Similarly, it is easier for a second language leaner to acquire vocabulary through the process of guessing from the context. In this sense, TV news can serve as a favorable factor. TV news is about our everyday life and students can always access news messages through other media in the form of their mother tongue. They may have watched or read the same piece of news from a local TV news programme or a local Chinese newspaper. With the background knowledge of certain pieces of news, they can predict the content and guess the meanings of the unknown English words from context. Gass and Varonis (1984) provide evidence that familiarity with the topic facilitates comprehension of nonnative speech. Oxford and Scarcella (1994) believe that by far the most useful strategy for vocabulary learning is guessing from context. Beheydt (1987) left no doubt as to his opinion: "From a psychological as well as a linguistic point of view, undeniably the first guideline would be that vocabulary must be learned in context. The meanings of words are more easily semanticized if they are embedded in a meaningful context."

Similarly, Huang and Eskey's (2000) study investigated the effects of closed- captioned TV (CCTV) on the listening comprehension of intermediate ESL students. Their study revealed that captions improved not only listening comprehension skills of college-level ESL students but also their general comprehension and vocabulary development. In another study on the effects of captions, Markham (1999) investigated the effects of captioned videotapes on second language listening word recognitions skills. He reported that the presence of captions significantly improved the adult ESL learners' listening word recognition of English language regardless of the level of pictorial support of the video. Another line of studies investigated the effects of captioned and subtitled TV programs on the acquisition of vocabulary (Goldman \& Goldman, 1988; Koolstra \& Beentjes, 1999; Koskinen et al., 1985; Markham \& Peter, 2003; Neuman \& Koskinen, 1992).

Koolstra and Beentjes' study focused on elementary-level Dutch-speaking students, and investigated the level of improvement in reading vocabulary knowledge by watching Dutch- subtitled English language television programs at home. In another study, Neuman and Koskinen's middle school-level ESL students increased their English language reading vocabulary knowledge significantly after lengthy exposure to the target language captions. Both of these studies involved the use of extended exposure to the target language videos with captions and/or subtitles, and yielded favorable implications about the effects of captions. In another study, Koskinen et al. chose their participants from the residents of a correctional facility. Many researchers like Snyder and Colon (1988) found that it is one of the good means to expose students to authentic language use in context. "TV offers visual and audio clues to meaning. The learner just hears the language but sees the context in which it is used. The learner can see in what kind of situation a person is addressed formally or informally and why. In other words, TV brings the outside world into the classroom. It gives the class and the teacher something to talk about, beyond the confines of the classroom. In this sense it can be a powerful stimulus to communication in class."

In fact, there are many other researches that have confirmed that extra-linguistic information can have dramatic effects on comprehensibility. In a critical analysis of L2 vocabulary learning techniques, Oxford and Crookall (1990) stated that "most learners are capable of associating new information to 
concepts in memory by means of meaningful visual images that make learning more efficient. Visual imagery is known to help learners to package information more efficiently than they would if using just words alone. Moreover, the pictorial-verbal combination involves many parts of the brain, thus providing greater cognitive power."

A study by Snyder and Colon (1988) investigated the influence of both audio and visual aids on facilitating second language acquisition. Two groups were taught for 7 weeks under two different conditions. One was exposed to a standard curriculum with audio-visuals limited to overhead transparencies, audiotapes with accompanying fill-in pictures, and slides. The other group was exposed to a curriculum enriched with more audio-visual aids, such as additional overhead transparencies, audio tapes and slides, as well as one bulletin board for reviewing material, and another for students to bring in materials from home, pictures from magazines, cut-out dolls to identify and reinforce names for parts of the body, and picture flash cards.

After testing both groups on the material covered, it was found that the group provided with additional audio-visual aids performed significantly better in vocabulary retention. Duquette and Painchaud (1996) compared listening to a dialogue with and without visual aids in order to determine which would better allow learners to guess the meaning of new words. Participants listened to a dialogue on the subject of driving a car under two conditions. Under the first condition, they listened to a dialogue while a videotape played. Under the second condition, they listened to the same dialogue with only an audiotape. Results indicated that the learners in the video group made gains on 8 unfamiliar words out of a total of 40, whereas the audio- only group learned only 3 . The authors speculated that the "match between prominent visual cues and linguistic ones allows for the inference of unfamiliar words." Regarding motivation the study found that students need the willingness to be active learners over a long period of time, for without this, they are unlikely to achieve any substantial vocabulary size, regardless of the quality of instruction. (Schmitt, 2008) A study conducted by Zhang (2009) investigated the foreign language vocabulary learning situation of 481 undergraduates in terms of their perspective of vocabulary learning, strategy use and vocabulary size. Based on the questionnaire investigation and vocabulary level tests, the most frequently used strategies include dictionary use, guessing the meaning and note-taking.

The undergraduates hold the perspective of learning vocabulary in the process of using them in contexts and do not agree with that of learning vocabulary only by rote memorization. He suggested that teachers should encourage students to learn to se lect and use certain learning strategies according to their own cognitive styles or personal preferences. According to the Input Hypothesis proposed by Krashen (1985), one of the most prominent theories of second language acquisition, language acquisition focuses on comprehensible input which causes language acquisition. He argued it is essential not to focus on explicit grammatical structures or learning activities but rather to occupy classroom time with acquisition tasks or activities. He believed that teachers should provide the students with extra-linguistic information, context and knowledge of the world to help understand language that contains structures a bit beyond their current level of competence. Nothing but multimedia technology can meet these requirements. Through watching English news, students can make use of images on videos and their background knowledge to understand the messages and acquire the language.

\section{Mate rials and Methods}

The study subjects of this are undergraduate students at College of sciences and Arts - Arrass, Qassim University. The subject are currently level three students taking a course known as "Reading and vocabulary building 3. The course book taught is "More Reading Power, Second Edition, Mikulcky, B.\& Jeffries ,L.( 2004). The subjects are divided into two groups experimental group and control group. Pre-test and post-test based on three questions was conducted and distributed to (50) subjects. The test was standard one with a variety of questions such as matching, multiple choice and fill in blanks. Afterdata collection the researchers used office excel to analyze the data.

\section{DATa ANALYSiS AND Discussions}

The data analysis of the pre-test and post-test for both groupsshows that in the pre-test ,the majority of the students find it difficult to guess the meanings of words that are mainly used in specific situations 
The Impact of Using English Medium Networks Breaking News on Enriching EFL Learners' Vocabulary. A Case Study of the Saudi University Students

and crises such as flood and an earth quake. For instance, most of the subjects failed to use calamity, disaster, destruction and floods correctly, although they have been taught how to scan, preview, skim and guess. While in the post-test there is a good progress in the experimental group as a result of exposing students to the English language breaking news. Thus, the researchers think that it is extremely important to encourage students to be access to breaking newsprogrammmes so as to enrich their English vocabulary.

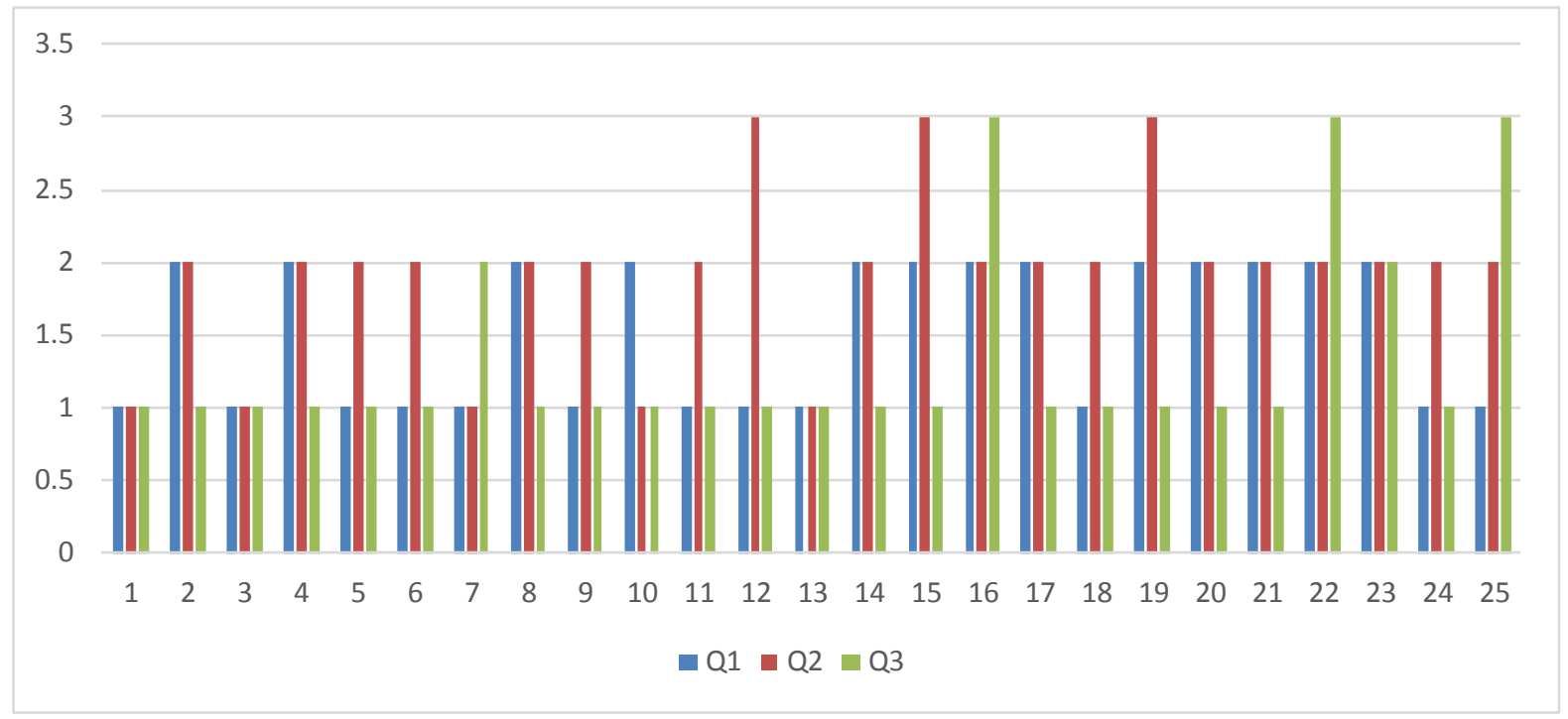

Figure1.The frequency distribution for the respondents' answers about the whole test questions 1,2,3.

The above figure shows the frequency distribution of the test questions given to the study subjects. The figure illustrates that the majority of the students have completed the task successfully. It is also remarkable that items no $(16,22,25)$ received the highest percentage of conformation. Simply because the subjects found it easier to classifiedboth regular nouns and verbs successfully. On the other hand, the figure also illustrates that the subjects found it difficult to classify the irregular verbsas well as some irregular nouns as shown in question no 3 .

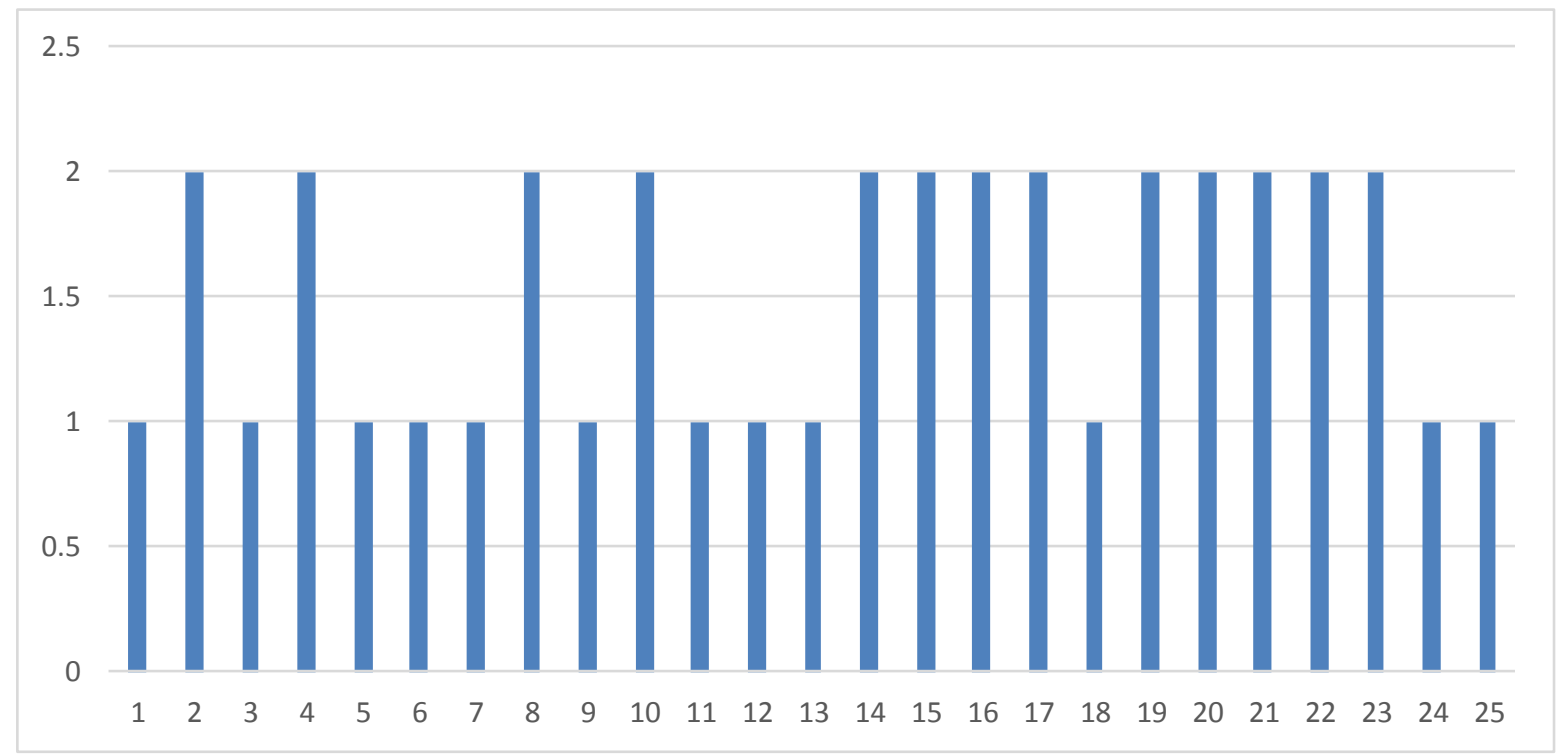

Figure2. The frequency distribution for the respondents' answers about question 1.

Figure no.( 2) illustrates that the respondents' answers about the first question, where the subjects were asked to match (A) with (B). It is significant that the subjects found it difficult to match words such as "flood" with the accurate definition in column B. The researchers believe that the course book taught to the subjects is not rich enough with such kind of vocabulary. Therefore, the majority of the subjects failed to match them with their meanings. 


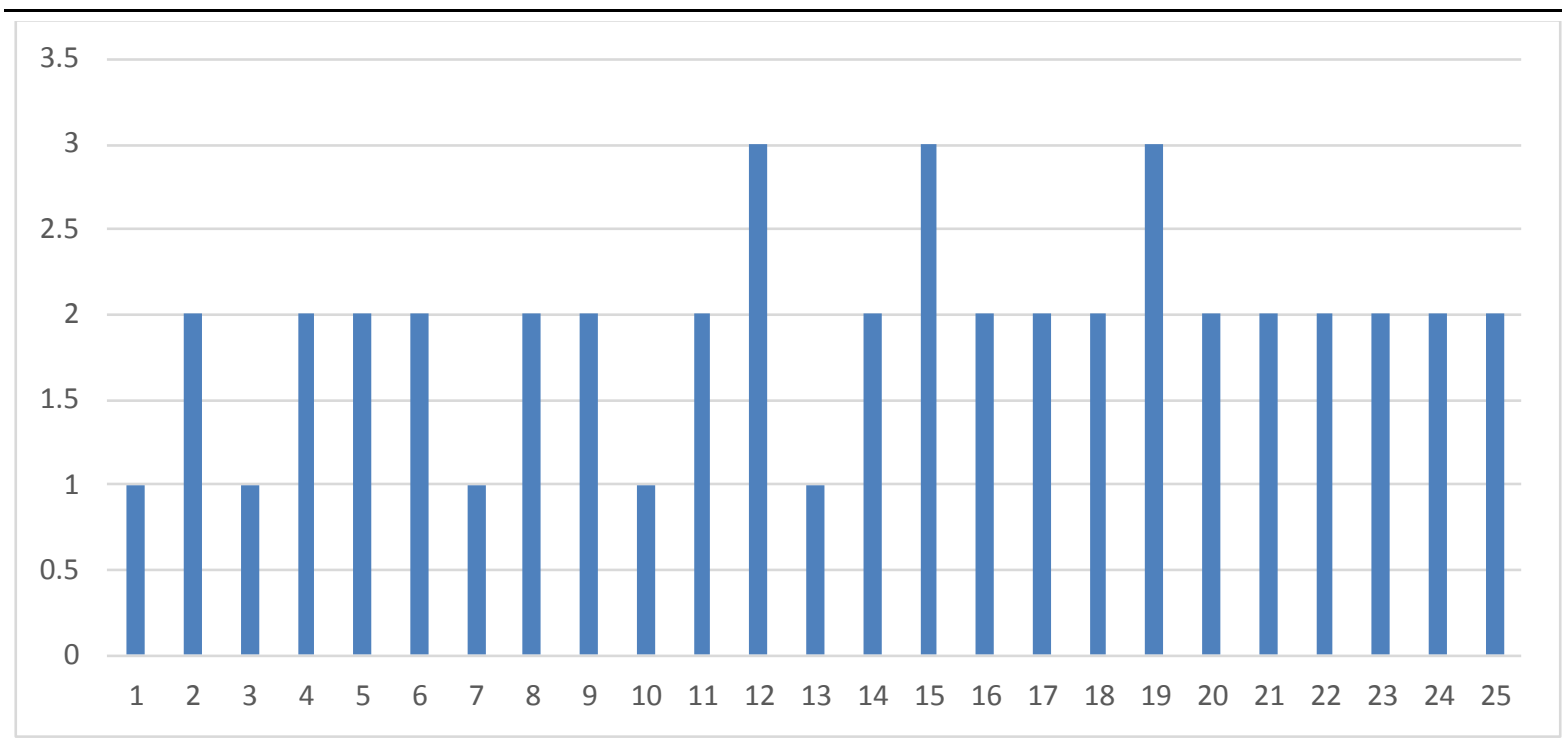

Figure3. The frequency distribution for the respondents' answers about question 2.

Regarding figure no. 3 it is remarkable that the majority of the respondents were able to fill the new words in blanks successfully. Moreover, the figure illustrates the difficulties faced by the subjects in items $(3,7,10)$, where they asked to fill words such as disaster and calamity in blanks. Thus, the researchers still believe that these words are not included in the course book taught to the subjects.

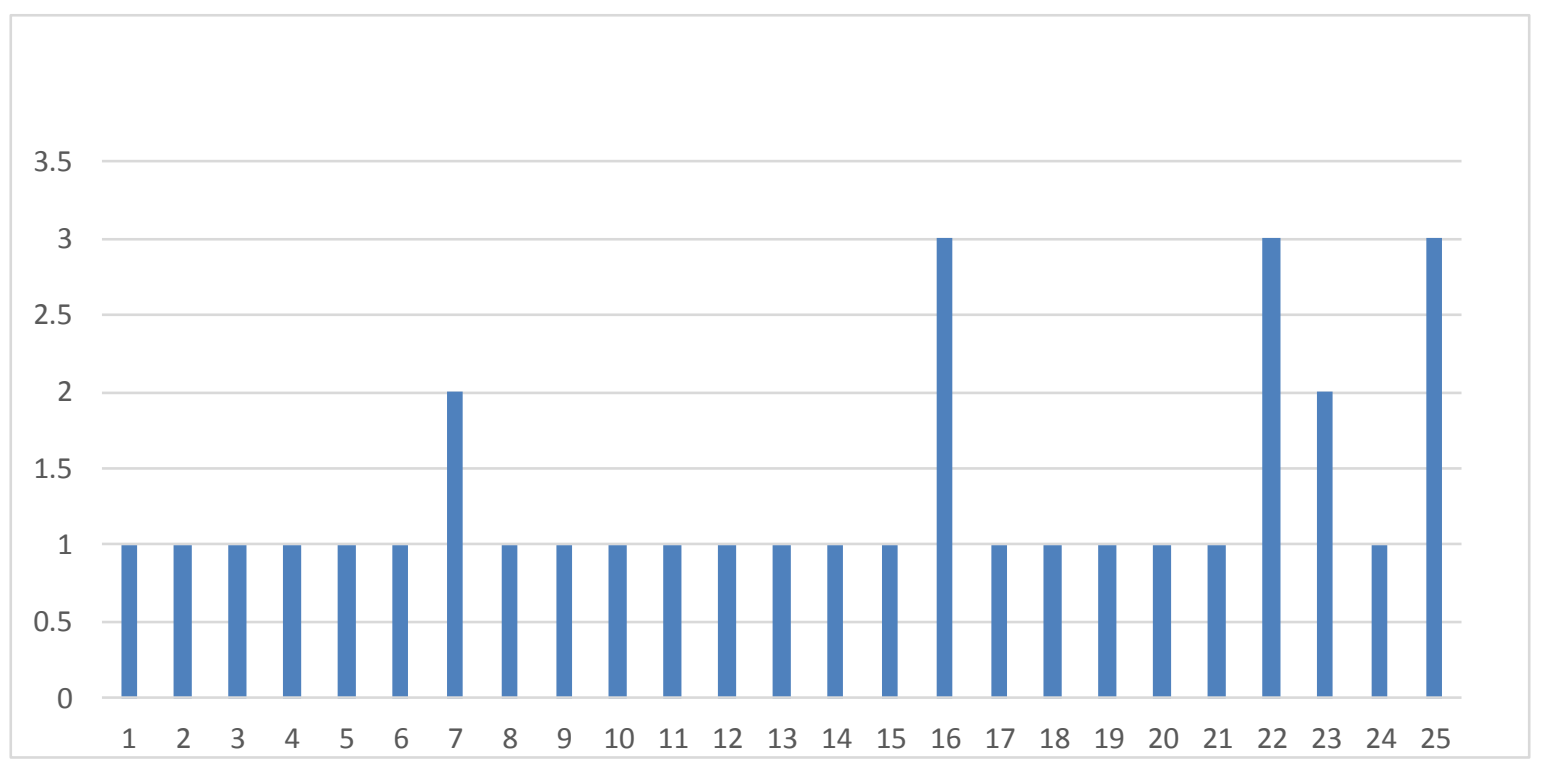

Figure4. The frequency distribution for the respondents' answers about question 3.

In question three, the subjects are given a number of words (verbs and nouns) and they asked to write $(\mathrm{N})$ for nouns and $(\mathrm{V})$ for verbs. The figure shows that majority of the subjects completed the task successfully with plural nouns. Whereas singular nouns were challenging to the subjects;for instance, they found it difficult to determine whether words such as (rice and snow) are nouns or verbs. Generally speaking, it is remarkable that the respondents are not able to distinguish both irregular verbs and nouns effectively.

\section{CONClusion}

This study and after the data were collected and analyzed the researchers arrived at the following findings:It has been found out that the course book is the only resource of learning and teaching vocabulary to undergraduate EFL Students. The findings reveal that being access to English TV breaking news facilitates enriching vocabulary building. Using English network channels in EFL classrooms also raises the learning motivation of the students. Hence it can be concluded that using English language TV. Breaking news is an effective method that can be used in EFL classes to enhance vocabulary development. Therefore, it is advisable to encourage EFL learners by using this method in different language skills development. 


\section{REFERENCES}

Herron, C. A., Hanley, J. E. B., \& Cole, S. P. (1995). A comparison study of two advance organizers for introducing beginning foreign language students to video. Modern Language Journal, 79(3), 387-395.

http://dx.doi.org/10.1111/j.1540-4781.1995.tb01116.x

Krashen, S. D. (1981). Second Language Acquisition and Second Language Learning. Oxford: Pergamon Press.

Koskinen, P. S., Wilson, R. M., Gambrell, L. B., \& Neuman, S. B. (1993). Captioned video and vocabulary learning: an innovative practice in literacy instruction. The Reading Teacher, 47(1), 36-43.

Nation, I. S. P. (2006). How large a vocabulary is needed for reading and listening. The Canadian Modern Language Review, 63(1), 59-82. http://dx.doi.org/10.3138/cmlr.63.1.59

Neuman, S., Burden, D., \& Holden, E. (1990). Enhancing children's comprehension of a televised story through previewing. Journal of Educational Research, 83, 258-265.

Schmidt, R. W. (1990). The role of consciousness in second language learning. Applied Linguistics, 11,129-158.http://dx.doi.org/10.1093/applin/11.2.129

Snyder, H., \& Colon, I. (1988). Foreign language acquisition and audio-visual aids. Foreign Language Annals,21(4), 343-384. http://dx.doi.org/10.1111/j.1944-9720.1988.tb01079.x

Schmidt, R. W. (1990). The role of consciousness in second language learning. Applied Linguistics, $11,129-158$.

http://dx.doi.org/10.1093/applin/11.2.129 